\title{
The reality of the quantum jump
}

Three-quarters of a century after the concept of quantum jump was introduced by Bohr, three independent measurements have emerged on each other's heels.

THE phrase "quantum jump" has long since escaped from physics; as a jump or, more dramatically, a "leap", the concept is applied in a variety of unconnected fields, from political upheavals to the emergence of new techniques in, say, modern dance. Quite properly, quantum jump has become a descriptor of discontinuity. So it may be of some general interest that quantum jumps, in the sense in which Bohr first used the term more than half a century ago, have just been observed for the first time.

Why should it have taken so long? The basic idea is that an atom capable of absorbing a light quantum of some determined frequency will, when it does so, jump into another state "instantaneously". The adverb of time is not to be taken literally, but guessed at from Heisenberg's uncertainty relations, but implies that the time taken to jump is a small fraction of the periodic time corresponding to the frequency of the radiation.

So the experiment to demonstrate that quantum jumps are real is simply designed: take a single atom, arrange that it absorbs a photon whose energy corresponds to the difference between one electronic state and another and then demonstrate that the atom does jump from one state to another as the photon is absorbed.

But only in the past few years have people been able seriously to think of "taking a single atom"; for several reasons, but chiefly because of the opportunities for accurate spectroscopy provided by welltuned laser beams, there has evolved a family of electromagnetic traps for single atoms. The other ingredients in this recipe for an experiment are not so simple either; demonstrating that there has been a quantum jump will simple-mindedly entail a measurement of some kind which, if badly planned, will disturb the state it is intended to demonstrate. So the three successful experiments now independently reported are technical as well as intellectual achievements.

The essence of the two experiments is the technique for showing that there have indeed been jumps, which appears most immediately to derive from a suggestion last year by Richard J. Cook and H. J. Kimble (Phys. Rev. Lett. 54, 1023; 1985), who in turn credit the University of Washington physicist Hans Dehmelt a decade earlier.

The trick is to choose an atom with an electronic state from which there are two well-understood transitions to two diffe- rent excited states, one of them readily accomplished (strong) and the other less so (weak). Irradiating a single atom with a laser whose frequency corresponds to the energy of the strongly-coupled transition has an easily predictable effect. The atom is repeatedly excited and, spontaneously, de-excited; the fluorescent radiation is emitted in all directions, and is not coherent with the laser beam, so that the atom is a powerful scatterer of radiation. Arranging that a single atom will scatter as many as $10^{\circ}$ photons a second appears not to be particularly difficult.

If the same atom is also irradiated with a laser corresponding to the energy difference of the weakly coupled transition, there will also be occasions when the atom is excited into the weakly coupled, not the strongly coupled state. And those occasions will be recognizable because the atom will no longer be fluorescent. Cook and Kimble were chiefly concerned to analyse the statistics of these occurrences, showing that they would form a pattern characteristic of Bohr jumping and also serve as a means of measuring the spontaneous transition probabilities between weakly coupled electronic states.

The three experiments now reported have the virtue of relying on two different atoms with very different transition schemes. A group from the University of Hamburg (Sauter, Th., Neuhauser, W., Blatt, R. \& Toschek, P.E., Phys. Rev. Lett. 57, 1696; 1986) have worked with singly ionized barium atoms, as have Warren Nagourney, Jon Sandberg and Hans Dehmelt (Phys. Rev. Lett. 56, 2797; 1986).

In each case, the competing weak and strong transitions are decays from an excited state to alternative de-excited states. But J. C. Bergquist, R. G. Hulet, Wayne M. Itano and D. J. Wineland (Phys. Rev. Lett. 57, 1699; 1986) from the US National Bureau of Standards at Boulder, Colorado, have worked with a set of three energy-levels of singly ionized mercury atoms in which the competing weak and strong transitions are excitations, replicating the case described by Cook and Kimble. The spectroscopic details are not strictly relevant to the essence of the result; all that matters is that the weak transition should, from time to time, temporarily sterilize the strong transition.

Inevitably, expectations are confirmed. The traces of the detectors measuring the fluorescence radiation are indeed interrupted from time to time, for periods whose duration is a measure of the strength of the weak transition. The three sets of experiments confirm that the time distribution of these intervals is exponential, as it should be. It is especially intriguing that, when two mercury ions are bundled together in the same electromagnetic trap, the fluorescence may occasionally be quenched not by the full amount but by exactly half, corresponding to a weak transition in only one of the ions.

At this late stage, with the notion of the quantum jump so firmly in charge of people's minds, the confirmation that quantum jumps actually occur is bound to seem a somewhat academic matter. But that entirely neglects the value of systems such as those developed for these measurements not merely in measuring the lifetimes of weakly interacting states (in which astrophysicists have a vivid interest) but of unravelling the various processes that contribute to the de-excitation of these states; in some circumstances, interatomic collisions matter most, in others, stimulation by radiation of the right frequency may determine the rate.

The way in which these experiments have evolved is, at the same time, a telling proof of the stimulus of new instrumental developments. Electromagnetic traps for single ions, or small numbers of them, were an obviously desirable technique five years ago. The spectroscopists were the chief customers . But the development of these instruments has lent credibility to the manipulation of single atoms, and the consequence of that has been a string of intriguing experiments in basic physies, the demonstration of quantum jumps among them. Perhaps the most intriguing so far is the demonstration, a few years back, that the transition probabilities between the energy levels of an atom placed in a conducting cavity are affected by the shape and size of the cavity, which is a direct but qualitative proof that quantum field theory is everything that people claim for it.

That, in all these cases, the direct demonstration of a principle follows by several decades the assumption that it is correct should not be a surprise. Most discoveries of principle, or at least the important discoveries, are inferences whose importance derives from knowing that demonstration is impractical. When the demonstrations can be carried out, the principles have been accepted. Galileo's alleged experiment at Pisa illustrates the point.
John Maddox 